\title{
INVESTIGACIONES
}

\section{La co-enseñanza desde enfoques inclusivos para los equipos del Programa de Integración Escolar}

\author{
The co-teaching from inclusive approaches \\ for the teams of the School Integration Program
}

\author{
Carlos Roberto Arriagada-Hernández ${ }^{a}$, Lorena Jara-Tomckowiack, \\ Oscar Ovidio Calzadilla-Pérez $z^{c, d}$ \\ ${ }^{a}$ Universidad Autónoma de Chile, Chile. \\ carlos.arriagada@uautonoma.cl \\ ${ }^{b}$ Fundación del Magisterio de la Araucanía, Chile. \\ lorena.jarat@fmda.cl \\ ${ }^{c}$ Universidad Católica de Temuco, Chile. \\ ${ }^{d}$ Escuela de Postgrados en Educación, Universidad Mayor, Temuco, Chile. \\ calzadilla1984@gmail.com
}

\section{RESUMEN}

En Chile los equipos del Programa de Integración Escolar tienen el desafío de empoderar a los docentes en la aplicación de la co-enseñanza como estrategia de trabajo colaborativo para la atención a la diversidad y la inclusión educativa. El estudio realizado se orientó hacia el análisis de la percepción de los docentes sobre las prácticas de los equipos multidisciplinarios para la implementación de la co-enseñanza en cinco establecimientos de la Fundación del Magisterio de La Araucanía ubicados en dos regiones del país. Se realizó una investigación cualitativa con el empleo de la entrevista y el análisis documental. Se obtuvieron cuatro redes semánticas y su interpretación arrojó inferencias sobre los factores que limitan o favorecen las prácticas de co-enseñanza, la inclusión educativa, el papel de la gestión directiva y su coherencia con los equipos del Programa de Integración Escolar, y la mejora de las prácticas en el aula.

Palabras claves: educación especial, co-educación, trabajo colaborativo, educación diferencial, formación de docente.

\begin{abstract}
In Chile, the teams of the School Integration Program have the challenge of empowering teachers in the application of co-teaching as a collaborative work strategy for attention to diversity and educational inclusion. The study carried out was oriented towards the analysis of teachers' perception of the practices of multidisciplinary teams for the implementation of co-teaching in five establishments of the Fundación del Magisterio de La Araucanía located in two regions of the country. A qualitative research was carried out with the use of the interview and the documentary analysis. Four semantic networks were obtained and their interpretation yielded inferences about the factors that limit or favor co-teaching practices, educational inclusion, the role of directive management and its coherence with the teams of the School Integration Program, and the improvement of practices in the classroom.

Key words: special education, co-education, collaborative work, teacher training.
\end{abstract}




\section{INTRODUCCIÓN}

La búsqueda de alternativas potenciadoras de escuelas y de buenas prácticas pedagógicoprofesionales inclusivas, conducen a resignificar los fundamentos y metodologías de la co-enseñanza. En este sentido la Organización de las Naciones Unidas (ONU), al examinar el Objetivo de Desarrollo Sostenible (ODS) en las sesiones del Foro Político de Alto Nivel celebrado en Nueva York (2019), ha llamado a garantizar una educación de calidad para todos. Al respecto connotó “... la necesidad de incorporar a más maestros a los sistemas educativos (...) Para que sus enseñanzas sean de calidad ellos mismos tienen que estar cualificados" (Agudo, 2019, p. 1). En relación lo planteado la atención de los niños con Necesidades Educativas Especiales (NEE) en contextos vulnerables, ocupa un rol de esencia en la consecución de las Metas Educativas 2030 (UNESCO, 2015) y los ODS (ONU, 2015) en los que la acción formativa de la escuela y sus profesionales los conducirá a la inclusión social y desarrollo pleno de su potencial.

El presente artículo surge como resultado de la investigación y la sistematización de profesional respecto al trabajo de los equipos multidisciplinarios de los Programas de Integración Escolar (PIE). En la actualidad este último concreta en la práctica las normas contenidas en la Ley de inclusión escolar (MINEDUC, 2015a) emitida por el Ministerio de Educación (MINEDUC) de Chile. En este contexto la co-enseñanza es una estrategia pedagógica pertinente a la gestión de la calidad de la educación. El estudio realizado se enfocó en el análisis de las prácticas del profesional encargado de coordinar el equipo PIE en cuanto a la implementación de la co-enseñanza en los establecimientos de la Fundación del Magisterio de La Araucanía (FMDA).

En la investigación realizada se comprobó la necesidad de identificar en las representaciones de los coordinadores PIE respecto a la inclusión, así como los elementos facilitadores y obstaculizantes de la co-enseñanza como alternativa de atención a la diversidad. En consecuencia, el objetivo de esta investigación radicó en: analizar las percepciones de los equipos multidisciplinarios del PIE respecto a la mirada inclusiva de la co-enseñanza dirigida a mejorar los aprendizajes de los estudiantes a nivel de aula en establecimientos de la FMDA, lo que deviene en desafío para la gestión directiva.

\section{SÍNTESIS SOBRE LA HISTORIA DE LA EDUCACIÓN ESPECIAL EN CHILE}

En Chile la aprobación de políticas públicas para la atención educativa y la integración sociolaboral prioriza la creación de oportunidades oportunas y enriquecedoras para el desarrollo pleno de niños, adolescentes y jóvenes (Rubio, 2017). Lo antes planteado sirve para justificar la creación de accesos y condiciones que ofrezcan respuestas educativas de calidad, ajustadas a la realidad de cada sujeto. A tales efectos uno de los desafíos socioeducativos más persistentes radica en la comprensión teórico-práctica de las NEE. El estudio de los antecedentes históricos de las NEE denota la prioridad inicial del aspecto clínico, de ahí su vínculo y fundamentación desde la Medicina y la Psicología.

Esto condicionó que la atención que se ofrecía en este nivel, hasta aproximadamente la década de los '60, fuese asociada con la cura o la corrección de una situación deficitaria o patológica, con marcado enfoque segregacionista. En este contexto la definición del 
principio de normalización por Wolfensberger (1986) sirvió para reforzar su visión integral en el abordaje de las NEE, al referir la utilización de medios culturalmente normativos (familiares, técnicas valoradas, instrumentos y métodos) para permitir que las condiciones de vida de una persona sean al menos tan buenas como las de un ciudadano medio.

El sistema educativo chileno ha progresado en dichas concepciones y construido en los docentes nuevos desafíos, de ahí que en las últimas dos décadas con los equipos PIE fortalecieran la búsqueda de estrategias psicopedagógicas de atención a la diversidad. En este contexto la aprobación de la Ley de inclusión (MINEDUC, 2015a) materializó con el Decreto 83/2015 (MINEDUC, 2015c), la aspiración de contar con aulas inclusivas para la atención de los niños con NEE; sin embargo, la sostenibilidad de este desafío tiene entre sus factores determinantes la calidad en la formación del profesional en cuanto al dominio de estrategias, el compromiso y la sensibilidad ante la singularidad y las particularidades de la educación de estos niños.

\section{PROGRAMAS DE INTEGRACIÓN EN CHILE}

La atención sobre las NEE constituye un reto y su evolución se ajusta al avance de la ciencia y a las continuas demandas sociales de las comunidades educativas. En Chile la reforma educativa de los años 60 se orientó, entre otros aspectos, a dar cobertura a toda la población en edad escolar. Este reto seguiría siendo enfrentado en las décadas posteriores sentando las bases para, en los años 90, institucionalizar los equipos PIE. En estos años se aprueban los Decretos $\mathrm{N}^{\circ} 1 / 98$ y $\mathrm{N}^{\circ} 1300 / 02$ en los que se enfatiza en la respuesta educativa a niños y jóvenes con NEE derivadas de una discapacidad o con Trastornos específicos del lenguaje (TEL) en la educación regular (Godoy, Meza y Salazar, 2004).

Las estrategias elaboradas para los equipos PIE tienen el propósito de mejorar los sistemas de ayudas y apoyos en el contexto del aula para atender a los estudiantes con NEE (de carácter transitorio o permanente). Esto sirve de fortalecimiento al carácter inclusivo y mediatizador de las relaciones sociales que a nivel aula experimentan los niños y jóvenes con NEE con la aspiración de lograr aprendizajes más conscientes y utilitarios (personales y sociales) en su trayectoria escolar e historia de vida.

En los años 90 toman auge las políticas para avanzar en la integración de niños con NEE en escuelas regulares y trabajar con los desafíos que esto implica. Más adelante, las acciones derivadas del Decreto $\mathrm{N}^{\circ} 170 / 2009$ sientan pautas en la institucionalización y comprensión pedagógica de las estrategias para la atención de estudiantes en una sala única por el espacio y diversa por sus sujetos que la integran.

Las Orientaciones Técnicas del Ministerio de Educación (MINEDUC, 2013) señalan la necesidad de que los equipos PIE a nivel de establecimiento fortalezcan las decisiones sobre: 1) el aprendizaje de los estudiantes; 2) el establecimiento de metas semestrales o anuales de aprendizaje; 3 ) el considerar la opinión, la participación y la colaboración respecto del currículo escolar; 4) la orientación e integración de la familia para alcanzar las metas de aprendizaje por los estudiantes; 5) la implementación de monitoreo, evaluación y seguimiento del progreso de los estudiantes; y, 6) la concreción de estrategias por curso para atender la diversidad. 


\section{TRABAJO COLABORATIVO Y CO-ENSEÑANZA}

Los cambios producidos en la atención de los estudiantes con NEE tienen entre sus propósitos lograr que estos reciban sistemas de ayudas y poyos por los docentes de aula y profesionales de los equipos PIE en el contexto de la sala común de clases u otros espacios, sin ningún tipo de discriminación debido a las particularidades de su individualidad. En este sentido el MINEDUC en Chile promueve normativas y orientaciones metodológicas para la funcionalidad práctica del trabajo colaborativo (como herramienta) y la co-enseñanza (como estrategia) en el aula.

En publicaciones consultadas el trabajo colaborativo se concibe como una estrategia de enseñanza-aprendizaje en la cual grupos de trabajo se organizan a partir de objetivos comunes definidos previamente, y sobre los que se organiza y ejecuta el trabajo a realizar (Angenscheidt y Navarrete, 2017; Sánchez, 2004; Temprado, 2009). Una de las peculiaridades del trabajo colaborativo radica en el involucramiento y nivel de participación de los participantes, aplicando procedimientos de enseñanza basados en la interdependencia, la interacción, la responsabilidad individual y la distribución de tareas. La conjugación de estas dimensiones es esencial en el alcance de las metas comunes intrínsecas al trabajo colaborativo.

En relación con lo expresado el trabajo colaborativo constituye una herramienta pedagógica para mejorar la calidad de los aprendizajes, y se ha definido como una metodología de enseñanza y de realización de la actividad laboral. Este se basa en el reconocimiento y creencia de que el aprendizaje y el desempeño profesional se incrementan cuando se desarrollan destrezas cooperativas para aprender y solucionar los problemas profesionales que la acción educativa demanda (MINEDUC, 2013).

Antes de la implementación del Decreto No. 170/2009 del MINEDUC existía evidencia de investigaciones desarrolladas en Chile en las que se concluía el débil dominio y empleo del trabajo colaborativo entre docentes. A tales efectos, la co-enseñanza como modalidad de trabajo colaborativo cobra relevancia para los docentes al conducir a la mejora mediante acciones profesionales de planificación, instrucción y evaluación colaborativa; todas ellas, en coherencia con las metas comunes y las necesidades de los estudiantes (Murawski, 2008; Rodríguez, 2014).

La dinámica de trabajo colaborativo implica acciones dentro y fuera del aula, por lo que combina competencias profesionales de enseñanza entre docentes, en el plano curricular, psicopedagógico, sociológico y clínico. También, involucra coordinar el trabajo hacia metas comunes, compartir un sistema de creencias, demostrar paridad en los roles, utilizar un liderazgo distributivo y actuar en forma cooperativa (Villa, Thousand y Nevin, 2008).

El concepto de co-enseñanza se enmarca según Cardona (2006) y Vance (2001) en tres categorías:

- Modelos de consulta, donde se caracteriza por el asesoramiento de un consultado, generándose jerarquía.

- Modelo colaborativo o de entrenamiento, el que nace de la insatisfacción del modelo de consulta, donde los apoyos son de manera igualitaria entre asesor y asesorado.

- La co-enseñanza, llamada también modelo colaborativo o de equipo.

En la consulta a la bibliografía especializada existe coincidencia en reconocer la aplicación de la co-enseñanza como una de las estrategias más pertinentes y viables para 
la inclusión en las condiciones de diversidad educativa que caracteriza el grupo escolar. Según Stuart, Connor, Cady y Zweifel (2007), entre las razones para implementarla se encuentran: la falta de comunicación entre pares, la coordinación entre profesionales, la fragmentación del currículum y la entrega de los apoyos a los estudiantes con dificultades de aprendizajes (Rodríguez, 2014).

Otras taxonomías de la co-enseñanza son expuestas en publicaciones de Friend et al. (2010), Hughes y Murawski (2001), y Villa et al. (2008), entre los que destacan:

- Co-enseñanza de observación, donde un profesor dirige la totalidad de la clase mientras el otro recoge información académica, conductual y social del grupo clase o de algunos estudiantes en particular.

- Co-enseñanza de apoyo, consistente en que un profesor toma el rol de conducir la clase, mientras el otro se rota entre los estudiantes, proveyéndoles apoyo individual, supervisando, recogiendo información y manejando la conducta.

- Co-enseñanza en grupos simultáneos, en que los educadores dividen la clase en dos grupos, a los cuales enseñan en forma paralela.

- Co-enseñanza de rotación entre grupos, en la que los profesores trabajan con grupos diferentes de estudiantes, los docentes se rotan entre grupos y uno de los grupos trabaja sin presencia continua del profesor.

- Co-enseñanza en estaciones, los docentes dividen el material y los estudiantes en estaciones que funcionan en forma simultánea. En el desarrollo de la clase los estudiantes rotan entre las estaciones, y una de las estaciones puede trabajar sin profesor.

- Co-enseñanza alternativa, ocurre cuando un docente trabaja con un grupo pequeño de estudiantes desarrollando actividades remediales, de preparación, enriquecimiento y evaluación, mientras el otro docente trabaja con la clase completa.

- Co-enseñanza complementaria, donde un profesor realiza acciones para mejorar o complementar la enseñanza provista por el otro profesor.

- Co-enseñanza en equipo, los co-educadores desarrollan simultáneamente la clase, alternándose los roles al conducir y apoyar la clase.

Estas taxonomías denotan las formas de abordar la ejecución de una clase, generar la co-enseñanza y producir aprendizajes significativos en los estudiantes. El MINEDUC (2013) reconoce cuatro enfoques de co-enseñanza, los que se originan en virtud del grado de confianza y aprendizaje que presenten los profesionales en dicho proceso, estos son:

- Enseñanza de apoyo: un docente asume el rol de líder, mientras el otro circula por la sala de clases prestando apoyos a los estudiantes.

- Enseñanza paralela: dos o más profesionales trabajan con grupos en diferentes secciones de clases, los co-enseñantes rotan en la sala de clases. Este enfoque lo utilizan los profesionales que están aprendiendo a trabajar juntos.

- Enseñanza complementaria: dos docentes aportan al proceso de la co-enseñanza, cada uno cumple un rol distinto y complementa al otro.

- Enseñanza en equipo: dos o más personas hacen lo que el profesor de aula ha hecho siempre, que es planificar, enseñar y evaluar asumiendo la responsabilidad de los estudiantes; por tanto, comparten liderazgo y responsabilidades. 
Según Villa et al. (2008), entre las exigencias de la transferencia profesional de la coenseñanza se encuentran:

- Coordinar el trabajo para lograr metas en común.

- Compartir la creencia que sustenta la experticia del otro.

- Demostrar la paridad de roles de profesores y estudiantes.

- Distribuir el liderazgo y sus funciones.

- Realizar un proceso cooperativo que ponga en evidencia las habilidades personales, el monitoreo de los avances y el compromiso.

- Carácter de voluntad, que evidencie las acciones de apoyo del equipo.

Las taxonomías de implementación de la co-enseñanza no son excluyentes entre sí, por el contrario, su validación en la práctica corrobora la necesidad de su complementariedad. El empleo de una u otra modalidad responde a las necesidades formativas de los estudiantes y la maestría pedagógica de los docentes en el logro de un enfoque participativo-dialógica de la co-enseñanza.

En Chile el MINEDUC (2013) enfoca la co-enseñanza en términos de acción, que implica a dos o más profesionales que comparten la responsabilidad de planificar, enseñar y evaluar desde el enfoque de reconocimiento y respeto a la diversidad a los estudiantes del salón de clases.

La co-enseñanza como estrategia no se circunscribe al acto o proceso de enseñar, su espectro pedagógico trasciende a la gestión del currículum. Esta afirmación es coherente con los criterios de Rodríguez (2012), al respecto el contenido en la Tabla 1 propone dimensiones y subdimensiones de la co-enseñanza y la gestión curricular.

Tabla 1. Dimensiones y subdimensiones de la co-enseñanza en la gestión curricular

\begin{tabular}{|l|l|}
\hline \multicolumn{1}{|c|}{ Dimensión } & \multicolumn{1}{c|}{ Sub-dimensión } \\
\hline \multirow{5}{*}{ Planificación de la enseñanza } & 1. Distribución de roles y responsabilidades. \\
& 2. Planificación general del aula común. \\
& 3. Diseño de las adaptaciones curriculares. \\
& 4. Determinación de las estrategias didácticas. \\
& 5. Planificación del enfoque de co-enseñanza. \\
& 6. Preparación de recursos. \\
\hline \multirow{5}{*}{ Didáctica del aula } & 1. Enfoque de co-enseñanza \\
& 2. Estrategias didácticas utilizadas. \\
& 3. Manejo de la conducta de los estudiantes. \\
& 4. Comunicación entre docentes. \\
& 5. Relación entre actividades de aula común y de aula de recursos. \\
\hline \multirow{5}{*}{ Evaluación } & 1. Diagnóstico de los aprendizajes previos. \\
& 2. Diagnóstico de las NEE. \\
& 3. Evaluación de proceso. \\
& 4. Evaluación final. \\
& 5. Evaluación de la co-enseñanza \\
\hline
\end{tabular}

Fuente: Elaboración propia, con ajuste a los criterios de Rodríguez (2012). 
Finalmente, la mejora continua de la calidad de la educación es uno de los desafíos de las políticas y prácticas educativas en Chile, lo que se enfoca en el reconocimiento de la diversidad, la inclusión de los estudiantes con NEE y la creación de oportunidades basadas en la equidad. En este contexto uno de los desafíos radica en formar a los docentes en el dominio teórico y práctico de estrategias para la atención de la diversidad en condiciones de inclusión educativa, como lo es la co-enseñanza. En relación con lo expuesto se valoró pertinente la necesidad de establecer referentes respecto al trabajo de los equipos PIE en los establecimientos escolares, por ello el análisis que se presenta sobre la gestión directiva.

\section{LA GESTIÓN DIRECTIVA RESPECTO AL TRABAJO DE LOS EQUIPOS DEL PIE}

En el desarrollo de esta investigación se indagó sobre las representaciones sobre gestión directiva en los docentes y su relación con la calidad en los aprendizajes de los estudiantes en contextos inclusivos. Tal como indica Weinstein (2002), no solo debe existir la mirada administrativa y formal en una organización, sino también de la gestión en relación con los recursos, las personas, sus procesos, el entorno y los resultados. Desde esta perspectiva la escuela es también una organización y hacer gestión en ella apunta a la necesidad de innovar para transformar (Leal-Soto, Albornoz y Rojas, 2016), y construir liderazgos (Muñoz y Marfán, 2012; Ulloa, Nail, Castro y Muñoz, 2012) como tendencias actuales, lo que lejos de constituir una preocupación es un estilo de trabajo enfocado hacia metas compartidas. En el caso de la educación entre los resultados que evidencian una gestión directiva de calidad lo constituye el desarrollo de actitudes, habilidades y capacidades de los estudiantes, y su reflejo en los aprendizajes escolares y para la vida.

En Chile la gestión directiva de los en las escuelas tiene puntos de contacto en sus accionar con la administración municipal o privada de establecimientos, y estos últimos se clasifican según sus sostenedores (privados, estatales o privados subvencionados). El MINEDUC promulgó de forma intencional la necesidad de guiar las prácticas de directores de escuelas y liceos para implementar los sistemas de gestión escolar mediante el Marco para la Buena Dirección (2005). Este último tiene como fortaleza el ordenamiento de la función directiva en cuatro dimensiones: liderazgo; gestión curricular; gestión de recursos; y gestión del clima y la convivencia. En la investigación de Muñoz y Marfán (2012) se defendió que en el caso chileno las políticas orientadas al liderazgo directivo escolar han desarrollado incipientemente las temáticas de la formación y desarrollo profesional de los directivos. Posteriormente a los criterios ministeriales de tipo normativo y metodológico sobre la buena dirección se integra al nuevo marco el liderazgo escolar (MINEDUC, 2015)

Respecto de la relación entre gestión y liderazgo Leal-Soto, Albornoz, y Rojas (2016) defienden la premisa de que la relación entre liderazgo e innovación ha sido poco estudiada en el contexto chileno. Dentro de los elementos de gestión identifican al liderazgo, y describen tres tipos con efectos diferentes: transaccional, transformacional y pasivoevitativo. Lo expresado evidencia también el carácter integrador y multidimensional de la gestión directiva.

Según Horn y Marfán (2010) las prácticas de liderazgo afectan de manera directa en la motivación, habilidades y condiciones de trabajo (a nivel de escuela y de aula) de los profesores, lo que se traduce en un mejor o peor desempeño docente, el cual a su vez afecta la calidad del aprendizaje de los estudiantes. Siguiendo a Leithwood, Day, Sammons, 
Harris, y Hopkins (2006), estas prácticas básicas y comunes de un liderazgo eficaz se agrupan en cuatro grandes dimensiones:

- Establecer dirección: proporcionar un propósito de carácter moral, que sirva de motivación para el trabajo del staff y los incentive a perseguir sus propias metas.

- Rediseñar la organización: establecer condiciones de trabajo que le permitan al personal el mayor desarrollo de sus motivaciones y capacidades.

- Desarrollar personas: construir el conocimiento y las habilidades que requiere el personal para realizar las metas de la organización, así como también, el compromiso y resiliencia, que son las disposiciones que éste necesita para continuar realizándolas.

- Gestionar la instrucción: gestión de prácticas asociadas a la sala de clases y supervisión de lo que ocurre en la sala de clases.

Además, Raczynski (2002) refiere que existen factores críticos de la gestión de calidad entre los que pondera a la labor educativa, y pueden asociarse a mejores resultados pedagógicos, entre ellos:

- Una escuela con sentido compartido de misión, que recoja de manera preferente el tema pedagógico, la formación y aprendizaje de los estudiantes, y el empoderamiento de docentes, padres y apoderados de la comunidad educativa.

- Liderazgo del director o equipo directivo, legitimado por la comunidad escolar.

- Trabajo en equipo entre directivos y docentes, y un buen nivel de compromiso de los profesores por el trabajo colectivo.

- Proceso de planificación institucional participativo.

- Participación efectiva de los distintos actores del sistema educativo.

- Clima laboral y de convivencia positiva entre docentes, entre directivos y docentes, y alumnos.

- Adecuada inserción del establecimiento con su entorno. (Weinstein, 2002, pp. 5657)

Finalmente, la gestión de los centros educativos de la FMDA se corresponde con el Modelo de Gestión de la Organización definidos en su Proyecto Educativo Institucional (PEI) (FDMA, 2014). Este aspecto revela la coherencia teórica de la primera respecto a la gestión directiva. De hecho, dicha institución defiende entre sus ejes estratégicos el modelo de gestión escolar los siguientes: la participación de los actores de la organización con enfoque en la calidad; la vinculación de la Fundación y participación en entornos y contextos de desarrollo; y, el aseguramiento de la calidad integral (FMDA, 2014, p. 24). Por todo lo antes expuesto, las acciones de los equipos PIE respecto a la co-enseñanza, se insertan en los proyectos educativos institucionales de los establecimientos, según el contexto sociocultural e institucional donde están insertados, y en su esencia son dinamizados por la gestión directiva escolar. 


\section{METODOLOGÍA}

\subsection{TIPO DE ESTUDIO Y OBJETIVOS}

El estudio asumió un enfoque cualitativo de investigación, de tipo exploratorio con un diseño de casos múltiples y enmarcado en una perspectiva hermenéutica de interpretación del objeto, al develar las percepciones de los docentes se decidió interpretar comprensivamente el discurso hablado de los sujetos (Cárcamo, 2005). El objetivo general de la investigación radicó en: analizar la percepción de los docentes respecto a las prácticas de los equipos multidisciplinarios para la implementación de la co-enseñanza en establecimientos con PIE de la FMDA; a tales efectos se precisaron objetivos específicos, entre los que se connota determinar factores o elementos facilitadores y obstaculizantes respecto de:

- el desempeño profesional de los coordinadores de los PIE respecto de la implementación de la co-enseñanza, en su relación con la manifestación de prácticas de educación más inclusivas.

- las prácticas de gestión pedagógico-curricular de equipos multidisciplinarios PIE, respecto de la implementación de la co-enseñanza.

\subsection{POBLACIÓN Y MUESTRA}

La población a los efectos de un estudio de tipo múltiple se conformó por cinco establecimientos de Educación Básica pertenecientes a la FMDA, la cual es colaboradora del MINEDUC en Chile. Esta Institución es de carácter particular subvencionado, y en su matrícula cuenta con estudiantes desde el nivel Pre-básico hasta la Enseñanza Media (Científico Humanista y de Formación Técnico Profesional). La FMDA está ubicada, como oficina central, con dirección Villa Alegre \#917 en la comuna de Padre Las Casas, IX Región de La Araucanía, y se encuentra en funcionamiento desde el 3 de enero de 1937; por su misión se dedica a la educación y evangelización de niños, niñas y jóvenes.

La FMDA declara en su PEI, ejes que se centran en la educación de calidad basada en la pedagogía de Jesús, la inclusión social, la educación integral, y considera la normativa legal vigente. Los establecimientos de la Institución se ubican en sectores rurales y urbanos de la Región del Biobío, La Araucanía y Los Ríos, en su matrícula es alto el índice de vulnerabilidad según las normativas del Estado para la identificación de estos. El 19\% de los estudiantes provienen de familias de la etnia mapuche, de bajos ingresos económicos y de escolaridad de los padres, y apoderados. De ahí que predominen estudiantes que viven en zonas caracterizadas de alto riesgo, lo que constituye una debilidad para la formación de valores y generan escasas posibilidades de estimulación intelectual y afectiva de los niños de dicho contexto.

\subsection{MUESTRA}

La muestra se intencionó hacia los profesionales miembros de los equipos del PIE, y se incorporaron sus coordinadores. Estos últimos a partir de las Orientaciones Técnicas del año 2013 y la normativa del MINEDUC emitida en el año 2009, se sugiere integran los equipos de gestión, con el fin de ayudar y apoyar en la ejecución del PIE a nivel institucional 
Estudios Pedagógicos XLVII N ${ }^{\circ}$ : 175-195, 2021

LA CO-ENSEÑANZA DESDE ENFOQUES INCLUSIVOS PARA LOS EQUIPOS DEL PROGRAMA DE INTEGRACIÓN ESCOLAR

y de grupo. Es por ello, que la muestra quedó delimitada en cinco establecimientos, tal como se representa en la Tabla 2.

Tabla 2. Establecimientos educativos participantes de la investigación

\begin{tabular}{|l|l|l|}
\hline \multicolumn{1}{|c|}{ Establecimiento educativo } & \multicolumn{1}{c|}{ Comuna } & \multicolumn{1}{c|}{ Región } \\
\hline Escuela San Rafael Arcángel & Padre las Casas & IX Región de la Araucanía \\
\hline Escuela Vilcún & Vilcún & IX Región de la Araucanía \\
\hline Escuela Ramón Guíñez & Pucón & IX Región de la Araucanía \\
\hline Escuela San Sebastián & Puerto Saavedra & IX Región de la Araucanía \\
\hline Complejo Educacional San Sebastián & Panguipulli & XIV Región de los Ríos \\
\hline
\end{tabular}

Fuente: Elaboración propia.

\subsection{INSTRUMENTOS APLICADOS EN LA INVESTIGACIÓN}

Entre los instrumentos aplicados en esta investigación se encuentran: la entrevista semiestructurada y el análisis documental. Además, se empleó la matriz de observación de las prácticas con base en los Estándares Indicativos de Desempeño para los establecimientos educacionales y sus sostenedores de la Agencia de la Calidad de la Educación del MINEDUC. Este último se vinculó con la Ley de inclusión $\mathrm{N}^{\circ} 20.845$ (MINEDUC, 2015a), la normativa vigente en el área de Educación Especial y su Decreto $\mathrm{N}^{\circ} 170$ (MINEDUC, 2009), Orientaciones Técnicas (MINEDUC, 2013), las que permitirán relacionar los indicadores con un enfoque inclusivo de la educación y el concepto de co-enseñanza.

En relación con la creación del instrumento, este se divide en tres categorías, presentadas en la siguiente Tabla 3:

Tabla 3. Categorías objeto de estudio

\begin{tabular}{|c|l|}
\hline Codificación de las categorías & \multicolumn{1}{|c|}{ Categorías } \\
\hline A & Conocimiento técnico \\
\hline a.1 & Conocimiento sobre inclusión educativa \\
\hline a.2 & Conocimiento sobre PIE \\
\hline B & Conocimiento de co-enseñanza \\
\hline
\end{tabular}

Fuente: Elaboración propia. 
Cada categoría, contiene preguntas para la etapa de ejecución de la entrevista e integra la percepción y experiencia de los participantes en cuanto a la opinión sobre las categorías expuestos, con la finalidad de crear un ambiente de confianza y conversación guiada, la cual hará al participante expresarse sobre fenómeno de investigación.

\subsection{PROCEDIMIENTO DE RECOGIDA Y ANÁLISIS DE DATOS}

El análisis cualitativo del contenido se desarrolla en un procedimiento de análisis de texto y diferentes técnicas de aplicación: 1) definición del material, la selección de entrevistas o partes de estas relevantes para responder a la pregunta de investigación; 2) análisis de la situación de recogida de datos; 3) caracterización mediante la transcripción de los textos; 4) análisis para los textos seleccionados y su interpretación; y, 5) definición de la técnica analítica mediante el uso de Atlas.ti (V.7). Con este último se definen las unidades analíticas, que según Flick (2004) constituyen unidad de codificación y lo define como el elemento más pequeño del material que se puede analizar, la parte mínima del texto que puede entrar en una categoría.

\section{RESULTADOS Y DISCUSIÓN}

Mediante codificación abierta, axial y selectiva, a través del uso de Atlas.ti se presentan los análisis asociados a las categorías: conocimiento técnico de inclusión educativa y PIE, conocimiento de co-enseñanza. La Figura 1 representa la red semántica respecto al conocimiento técnico, y de inclusión educativa y del PIE.

Figura 1. Red semántica I respecto al conocimiento técnico, de inclusión educativa y PIE (categoría: coordinadores PIE)

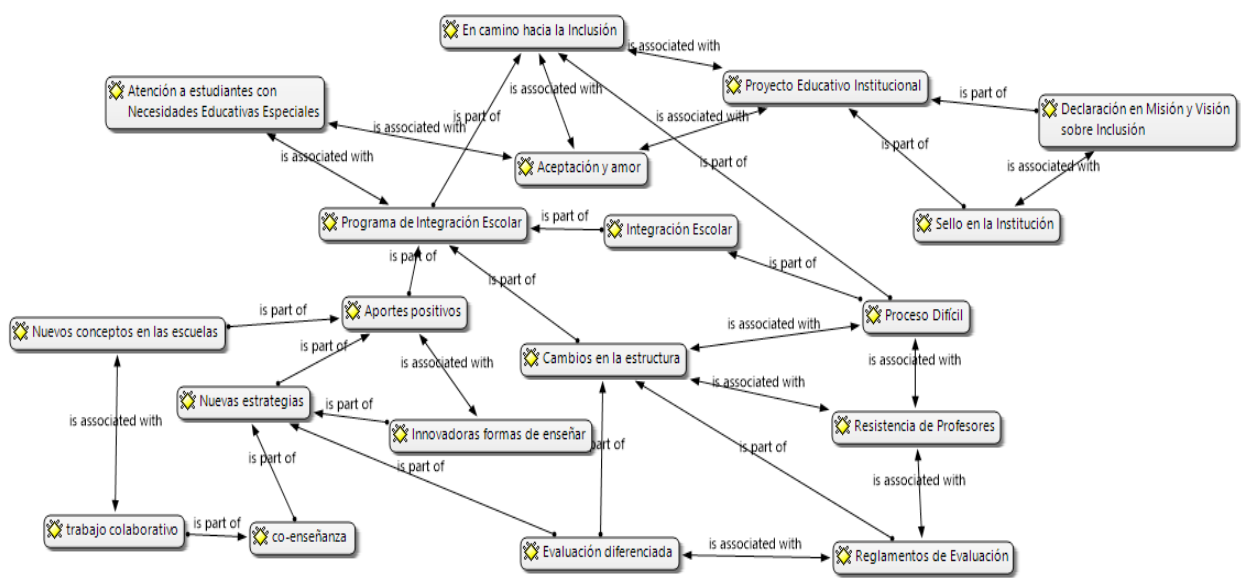

Fuente: Elaboración propia. 
Los profesionales en esta área de la investigación reconocen encontrarse en camino hacia la inclusión educativa. Además, se evidencia la identidad institucional de los establecimientos al declarar en los PEI de las comunidades educativas la importancia de la inclusión y la atención de los estudiantes con NEE. Esto último lo relacionan con el concepto de la inclusión educativa, y refieren que para ello es importante la aceptación y el amor que se pueda entregar, no solo a los estudiantes, sino además a sus familias que en muchas oportunidades no encuentran suficientes ayudas y apoyos.

La integración es la consecuencia del principio de normalización, el derecho de las personas con discapacidad a participar en todos los ámbitos de la sociedad con los apoyos que necesitan en el marco de las estructuras comunes de educación, salud, empleo, ocio y cultura, y servicios sociales; reconociéndoles los mismos derechos que el resto de la población. Asimismo, la integración educativa debe formar parte de una estrategia general, cuya meta sea alcanzar una educación de calidad para todos (UNESCO, 2001). Complementan las relaciones de percepción de la Figura 1 el contenido de las entrevistas, entre las que destacan:

E 4: "Inclusión educativa, es bien amplio y es difícil de definir, inclusión es aceptar la diversidad, diversidad no solamente relacionado a alguna necesidad, sino que necesidad educativa que es más bien relacionado con lo de educación, sino que considerando las características personales de todos, ya y ante eso es he un concepto amplio, hablar de inclusión es hablar de aceptar de poder o de tener la capacidad de ver las capacidades de cada una de las personas y abordarlo desde esa realidad, realidad que no existe hoy en día en nuestro sistema educativo, ya pero así lo veo, como una diversidad".

E 5: "Bueno, entiendo la inclusión educativa como la eliminación de barreras para acceder a los aprendizajes, ya sea con niños con o sin NEE".

E 8: "La inclusión va más allá de la integración que hemos conocido desde que empecé a trabajar, y antes por ende, es un paso más allá y tiene que ver con en que niños no solo con opción educativa especiales, sino que a todos los niños se le tomen en cuenta las diferentes características personales sus intereses, que estén todos en el mismo lugar y podamos dar respuestas a cada característica de cada niño".

Es por este motivo, que las Orientaciones Técnicas, emitida por el MINEDUC (2013), exponen la importancia de la sensibilización previa de la comunidad para la implementación de este tipo de programas, debido a que cuando esto se implementa de manera forzosa no genera los mismos efectos que genera en una comunidad de acuerdo y abierta a la atención a la diversidad [...] Constituye una herramienta para mejorar las relaciones interpersonales y el clima de convivencia escolar entre los estudiantes y distintos actores de la comunidad educativa. La decisión de implementar un PIE, requiere un análisis previo de su factibilidad y de un proceso de participación y sensibilización de la comunidad educativa, incluyendo a las familias y a los propios estudiantes, respecto del valor de la diversidad, sus aportes, implicancias, y de los roles que cumplirán los distintos actores en este proceso".

El cambio de estructura se basa en la reorganización de PEI, visiones y misiones que estén bajo el mismo lineamiento de la institución sostenedora, y en las normativas de evaluación del MINEDUC (MINEDUC, 2015c), los cuales contienen la incorporación de las nuevas exigencias y necesidades de todos los que desean y deben de aprender. 
En esta red semántica se evidencian los aportes del PIE de manera positiva e innovadora, debido a que trae consigo una propuesta a las comunidades educativas, que genera nuevos conceptos como lo son trabajo colaborativo y la co-enseñanza en el aula. En este sentido, una de las nuevas formas y estrategias de enseñanza lo constituye el Diseño Universal de Aprendizaje (DUA), el que se generó como código en la investigación y se incluyó en la familia de esta red semántica.

El DUA defiende la premisa de que la diversidad es la norma, no la excepción, por lo que las programaciones y las situaciones de aprendizaje se diseñan desde el principio ("a priori") para satisfacer las diferencias de aprendizaje, de manera que gran parte de las adaptaciones posteriores puedan ser reducidas o eliminadas. En relación sus principios, pueden ser aplicados en la planificación de las actividades para todos los estudiantes de una misma sala de clases, estos se enuncian a continuación:

- Proporcionar formas múltiples de presentación y representación, considera diversas modalidades sensoriales, estilos de aprendizaje, intereses, preferencias y múltiples inteligencias.

- Proporcionar formas múltiples y variadas de expresión y ejecución, supone diferentes formas de comunicación/expresión, y utilizar estrategias cognitivas y meta cognitivas, que permitan a los estudiantes responder según sus preferencias y estilo para aprender y demostrar lo que han aprendido.

- Proporcionar formas múltiples de motivación para dar respuesta a variados intereses y preferencias, consideran los principios de autonomía, motivación, personalización y variedad (CAST, 2008, pp. 5-6).

En relación con la co-enseñanza, la Figura 2 muestra la existencia de conocimientos sobre el concepto por los participantes (coordinadores de PIE), escaso respecto a los tipos de co-enseñanza aplicables en las aulas, como apoyo directo en los aprendizajes de calidad de los estudiantes.

Figura 2. Red semántica respecto al conocimiento de co-enseñanza (categoría Coordinadores PIE)

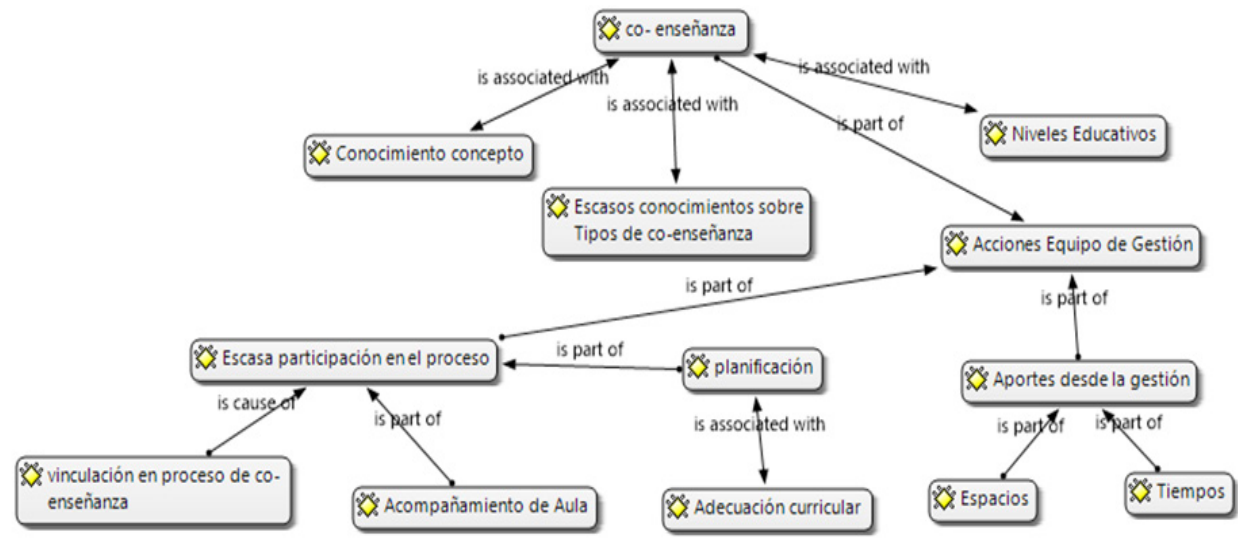

Fuente: Elaboración propia. 
En la red semántica se aprecia escasa participación en el proceso debido al bajo conocimiento en el área, e insuficiente nivel de acompañamiento de aula respecto al rol desempeñado. Se observa la generación de planificaciones y las adecuaciones correspondientes a casos particulares; sin embargo, desde el rol de coordinador PIE prevalece baja vinculación con el proceso y escasa participación para verificar su efectividad como estrategia de mejoramiento continuo.

Los criterios obtenidos en la entrevista complementaron, los resultados expuestos en la red semántica sobre el conocimiento de co-enseñanza al plantear:

\section{E 2: "Es cuando se está trabajando dentro del aula la educadora diferencial y la profesora de aula"}

E 14: "Sí he escuchado, pero no me recuerdo, son varios... siíyo sé, pero en este momento para decírtelo tengo la idea, pero yo sé que existen más".

E 4: "Sí, conozco pero bueno en relación a lo que establece el decreto no recuerdo exactamente los nombres, pero si he claramente hay niveles en los que inicialmente la co-enseñanza debería ser creo que están los apoyos, no recuerdo bien los nombres"... E 5: "Son tres niveles, el nivel de la evaluación el nivel de la planificación y el nivel... ¿cuál era el otro nivel? planificación, evaluación y la didáctica.

E 8: "Nunca me he aprendido los, las categorías, los como el nombre, pero te podría decir la definición..."

El resultado de esta red semántica muestra relación con lo aseverado en la obra de Rodríguez (2014) y Rodríguez y Ossa (2014), en la que se defiende que un aspecto clave para el éxito de las instituciones educativas es la planificación colaborativa, al generar espacios y tiempos para el trabajo colaborativo sistemático. Asimismo, es deseable que se realice un seguimiento de las experiencias de co-enseñanza, de modo que se valore su impacto en el aprendizaje de los estudiantes y el desempeño de los co-docentes. Además, se observó que desde los equipos de gestión se entregan las facilidades en los tiempos y espacios para generar la co-enseñanza; sin embargo, estos procesos requieren mayor elaboración por los equipos PIE y los profesionales que deben responder diariamente a estas necesidades. Es así, como lo indica Duk y Murillo (2011), con una visión más amplia del sistema de educación regular, los profesionales que trabajan en esta área deberían de recocer que aún carecen de herramientas para enseñar sobre la base de las diferencias, por lo que es un desafío y línea de trabajo.

La atención de la diversidad educativa es uno de los principios de sucesivas reformas que en la actualidad percibe un escenario socioeducativo más propicio para la integración de niños con NEE en la escuela regular. En contradicción con lo planteado sobre este principio los docentes de la Educación Básica, y los formados para la Educación Especial no cuentan con experiencia suficiente en el plano metodológico, sobre las prácticas de aula más adecuadas para atender la diversidad (Duk y Murillo, 2011).

Otro de los elementos objeto de estudio lo constituyó el liderazgo directivo, tal como se observa en la Figura 3, esta red semántica se asoció a las características de los directores de las comunidades. 
Figura 3. Red semántica respecto a liderazgo del director (categoría: Coordinadores PIE).

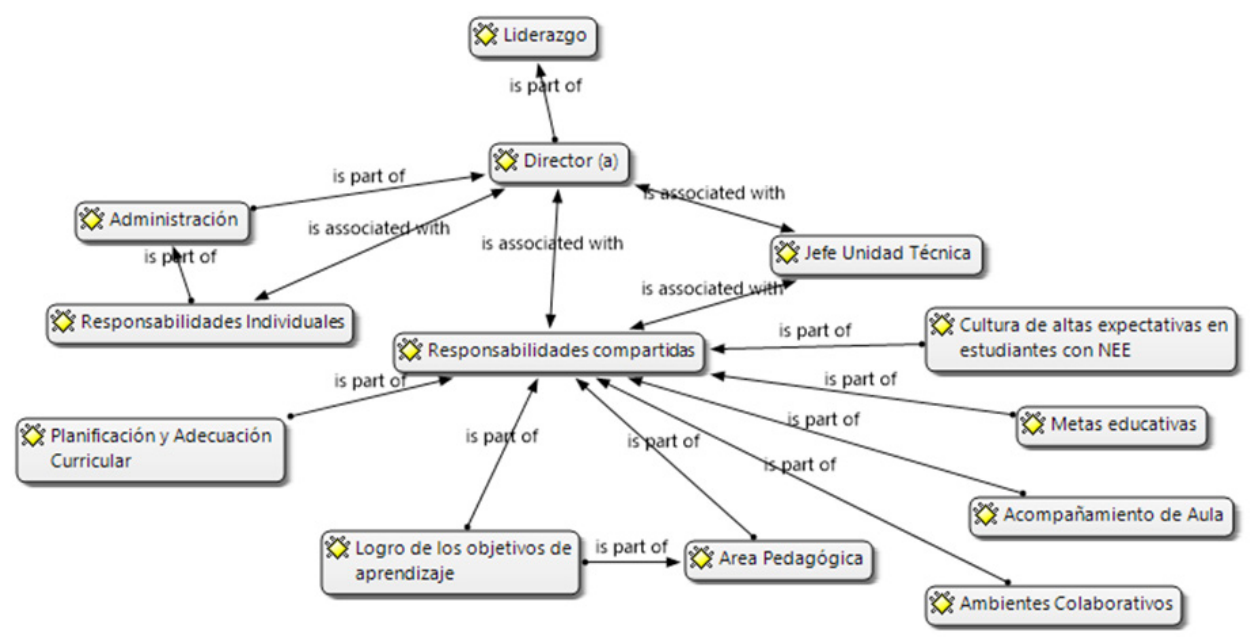

Fuente: Elaboración propia.

El contenido de la red semántica corroboró el carácter colaborativo de los profesionales para el trabajo con la co-enseñanza como estrategia para la atención de la diversidad y la inclusión educativa de estudiantes, con o sin NEE. Se ponderan las acciones de orientación pedagógica con responsabilidades compartidas para el equipo como los son: la cobertura curricular, el logro de los aprendizajes de todos los estudiantes, los ambientes colaborativos, el acompañamiento de aula, las metas educativas en relación con su creación e implementación de acciones para llegar a cumplirlas, la generación de una cultura con altas expectativas, entre otras.

La última red semántica presentada en esta investigación es que el área pedagógica, se relaciona al funcionamiento y cumplimiento de los equipos de gestión de los establecimientos, y da cumplimiento a la cobertura curricular y la coordinación de esta, evidenciando en casos específicos escaso tiempo para realizar el análisis en conjunto con los equipos.

La red semántica construida al respecto se ilustra en la Figura 4. 
Figura 4. Red semántica respecto a gestión curricular (categoría: Coordinadores PIE).

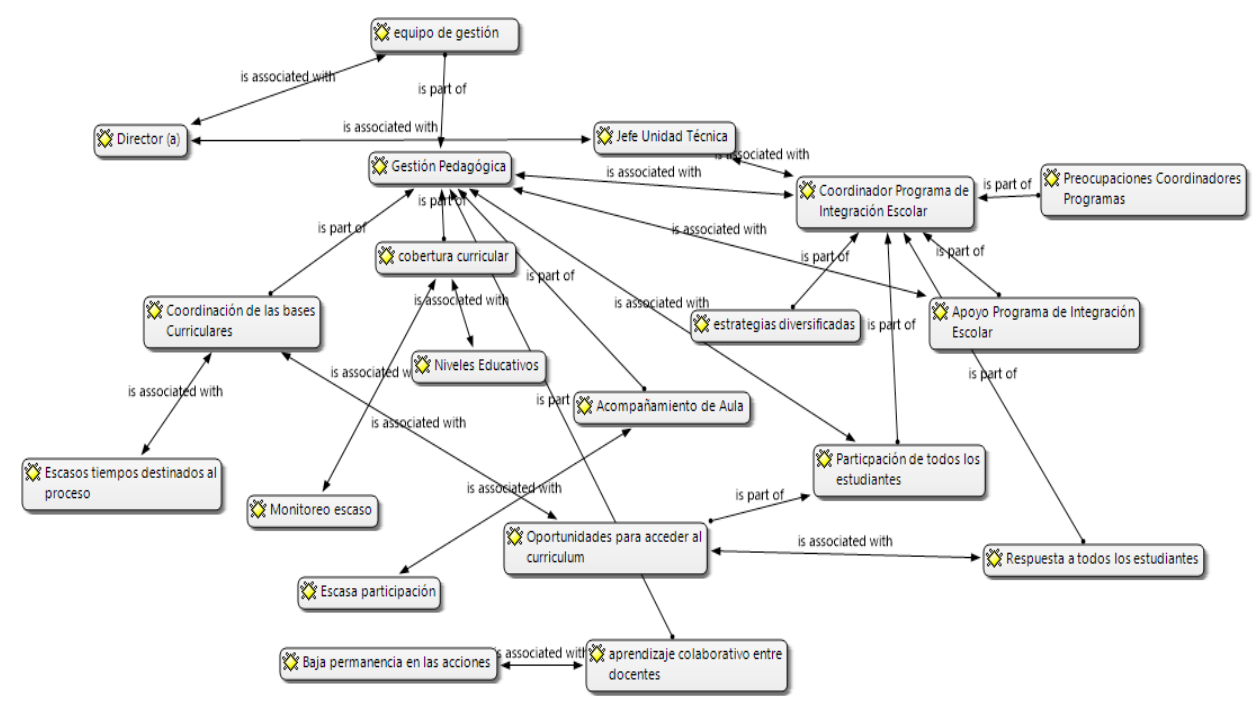

Fuente: Elaboración propia.

Esta red evidencia la delegación de funciones y roles, en las que el coordinador PIE se percibe como un facilitador del proceso y un puente de comunicación entre los equipos de aula y de gestión, y considera las propuestas curriculares y las acciones emanadas por los profesionales de apoyo.

Las redes semánticas demostraron la necesidad de compartir responsabilidades y trabajar de forma colaborativa en equipo. En relación con ello, el MINEDUC (2013) señala que existen diversas funciones que los coordinadores PIE deben realizar, por lo que no deja exento de responsabilidades a sostenedores, directores y jefes técnicos.

Entre las preocupaciones de los docentes, la familia y los apoderamos estuvo el ofrecer una atención educativa mediante la co-enseñanza que les permita los estudiantes acceder al currículo, y trabajar con los equipos de aula desde las actitudes y habilidades formadas en los estudiantes. Además, se ponderó la necesidad de diversificar las acciones de las estrategias, y personalizar en casos específicos en coherencia con los resultados del diagnóstico psicopedagógico y sus implicaciones en los procesos de planificación, enseñanza y evaluación; que respete el desarrollo psicobiológico y socioemocional del estudiante.

En el análisis general de los resultados se logró la construcción de cuatro redes semánticas en las que, si bien las opiniones son diversas y responden a la mirada del rol y función profesional específica en los establecimientos estudiados, es factible generar un análisis global de los resultados expuestos.

El modelo de trabajo inclusivo que se sistematizó en la investigación implica un trabajo más amplio, y una mirada de la educación desde la participación y la gestión de la calidad educativa para todos. La inclusión tiene como propósito eliminar barreras en todas las áreas y equilibrar las desigualdades, respetando la diversidad (UNESCO, 2001). El modelo de 
educación inclusiva compromete la educación de todos los niños, independientemente de la comunidad donde viven, etnia o lenguaje puedan aprender juntos, e incluye a los niños con NEE. Se trata de un modelo de escuela en la que no existen requisitos de entrada, ni mecanismos de selección o discriminación de ningún tipo, para hacer realmente efectivos los derechos a la educación, a la igualdad de oportunidades y a la participación.

En las entrevistas se confirmó que en establecimientos educativos específicos se evalúa y diagnostica centrado en la relevancia de las limitaciones para aprender y no en las competencias o potencialidades adquiridas, como vía para compensar y promover el desarrollo pleno (Calzadilla-Pérez, et al. (2017). En relación con esto Miranda (2013), plantea que lo que la sociedad debe igualar por encima de un umbral no son las oportunidades, sino las capacidades. En el terreno de la educación, ello implica una configuración mínima de recursos y capacidades aplicables en oportunidades escolares reales para aprender.

Con el fin de generar buenos procesos, desde las necesidades expuestas y los recursos establecidos se hace necesario el desarrollo del aprendizaje colaborativo. Según Zañartu (2003), este se centra básicamente en el diálogo, la negociación y en la palabra, en este caso específico, será desde la mirada de los docentes, y por supuesto aplicable en la acción educativa del aula.

En relación con los equipos directivos, y a la categoría liderazgo del director es que Bellei, Morawietz, Valenzuela, y Vanni (2015) sostienen que, no existe efectividad escolar sin equipos directivos sólidos y estables. Se sugiere en su publicación que el tema del liderazgo directivo sea asumido como política pública, donde se consideren elementos claves para directores y jefes técnicos como lo son: la identificación de los profesionales en relación con sus características y el desarrollo de sus capacidades y potencialidades. Con la salvedad de que esta limitación tiene respuesta en el Marco para Buena Dirección y el Liderazgo Escolar (MINEDUC, 2015b). Esto último, permitirá respaldar la importancia de mejorar los procesos de selección en el sistema público y privado de los lideres educativos. Además, la sostenibilidad del ciclo de mejoramiento continuo de los establecimientos depende, en lo fundamental, de la existencia de líderes escolares efectivos y procesos planificados de sucesión del liderazgo, que institucionalizan las prácticas, modos de trabajo, desarrollan el capital social y profesional de las escuelas.

Finalmente, los resultados, su análisis y discusión se corresponden con lo indicado en Chile como Marco para Buena Dirección y el Liderazgo Escolar (MINEDUC, 2015b). En este las prácticas de los directores de escuelas básicas se presentan como la principal fortaleza en la fijación de una dirección general de futuro en los establecimientos; sin embargo, la mayor debilidad reside en el menor tiempo y dedicación a la gestión de la enseñanza y el aprendizaje en establecimientos municipales y particulares. Los resultados de las redes semánticas construidas fueron complementados con las entrevistas y las observaciones, cuya triangulación permitió obtener evidencia científica de los sesgos que limitan a la coenseñanza y los desafíos que implica su empleo de esta en los establecimientos de la FMDA.

\section{CONCLUSIONES}

El origen de este artículo responde a la búsqueda de soluciones científicas mediante la investigación educativa en la problemática de aplicación de la co-enseñanza como estrategia de trabajo colaborativo para la atención a la diversidad en condiciones de inclusión educativa 
en establecimientos de la FMDA. Por lo que se profundizó en el estado actual y los desafíos que la co-enseñanza para los profesionales de los equipos PIE. La aplicación de instrumentos de investigación y su procesamiento mediante el software Atlas.ti condujo a la determinación de cuatro categorías: conocimiento técnico, de inclusión educativa, del Programa de Integración Escolar, y de la co-enseñanza.

No obstante, el estudio de los referentes históricos respecto a la Educación Especial chilena y los equipos PIE permitió profundizar en la pertinencia y actualidad de las políticas públicas al respecto. El análisis teórico del trabajo colaborativo y la co-enseñanza, y el papel de la gestión directiva acerca del trabajo de los equipos del PIE permitió inducir generalizaciones respecto a la vitalidad del tema objeto de investigación y los desafíos intrínsecos a su materialización en la práctica. A tales efectos se reconoce la necesidad de perfeccionar la explicación metodológica de la co-enseñanza y en su materialización la labor de los equipos PIE en coherencia con la gestión directiva a nivel de establecimiento educativo.

La interpretación de las redes semánticas construidas y su triangulación con los instrumentos aplicados permitió establecer generalizaciones sobre los desafíos actuales y sus implicaciones en los establecimientos educativos de la FMDA, entre los que se destacan:

- La gestión directiva y pedagógica debe sistematizar entre sus prioridades el dominio técnico de la co-enseñanza y su empleo como estrategia para promover buenas prácticas de inclusión en contextos diversos y vulnerables en los que se educan los niños de los establecimientos de la FMDA.

- El accionar de los coordinadores y equipos PIE para la implementación de la coenseñanza debe generar propuestas que vinculen la gestión directiva y educativa a nivel de establecimiento. Con ello es factible alcanzar mayores niveles de coherencia entre el desempeño profesional de directivos y docentes, las normativas del MINEDUC y el modelo de gestión de la FMDA.

- Respecto a la co-enseñanza se evidenció un dominio aún "epidérmico" de su concepto y su empleo como estrategia, que genera limitaciones para su empleo en el salón de clases.

- La gestión de los equipos PIE sobre la formación en inclusión educativa de los docentes debe fomentar las prácticas de atención al niño y las acciones de orientación (a la familia y apoderados) como evidencia de una escuela abierta, integradora y transformadora.

En el análisis de los factores facilitadores y obstaculizadores se determinó que directivos y equipos técnicos muestran compromiso con la atención a la diversidad aunque falta perfeccionar la dimensión pedagógica de las acciones. En la gestión pedagógicocurricular de directivos sobre la co-enseñanza se evidenció colaboración con los equipos PIE. Además, pese a las situaciones en las planificaciones y el agobio laboral, los profesores realizan sus programaciones y adecuaciones curriculares para atender a las necesidades de los estudiantes. De forma indistinta el trabajo colaborativo es asociado por algunos docentes a la acción de compartir materiales, y no a la práctica de intercambiar experiencias profesionales. Se requiere de la observación y análisis por los docentes de clases en las que se aplican estrategias de co-enseñanza. 


\section{REFERENCIAS BIBLIOGRÁFICAS}

Agudo, A. (2019, 11 de julio). La ONU alerta de una crisis global de aprendizaje. El País. Última visita 11 de julio de 2019. Recuperado desde https://elpais.com/elpais/2019/07/10/planeta_ futuro/1562751921_561796.html?fbclid=IwAR0NC1_TI6E6CC_wYZ3mrOoE9-O+++fzqyp xVpSFtFvsCPXj5MtYKsrW3NoULEyhash=bdd91c98-62da-4f2d-a960-4031764a11a5yutm_ medium $=$ socialyutm_source $=$ facebook

Angenscheidt, L. y Navarrete, I. (2017). Actitudes de los docentes acerca de la educación inclusiva. Revista Ciencias Psicológicas, 11(2), 233-243. doi: https://doi.org/10.22235/cp.v11i2.1500

Bellei, C., Morawietz, L., Valenzuela, J. y Vanni, X. (2015). Nadie dijo que era fácil. Escuelas efectivas en sectores de pobreza, diez años después. Santiago: Lom Ediciones. Última visita 12 de noviembre de 2019. Recuperado desde http://repositorio.uchile.cl/handle/2250/136719

Calzadilla-Pérez, O., Ponce, S. y Salazar, M. (2017). La estimulación del talento verbal en la escuela primaria. Transformación, 13(2), 192-210. Recuperado desde http://scielo.sld.cu/scielo. php?pid=S2077-29552017000200004yscript=sci_arttextytlng=pt

Cárcamo, H. (2005). Hermenéutica y análisis cualitativo. Cinta de Moebio. Revista de Epistemología de Ciencias Sociales, 23, 204-2016. Recuperado desde http://www2.facso.uchile.cl/ publicaciones/moebio/23/carcamo.htm

Cardona, M. (2006). Diversidad y educación inclusiva. Madrid: Pearson Educación.

Center for Applied Special Technology (CAST). (2008). Universal design for learning guidelines (Version 1.0.). Wakefield, MA: Author. Última visita 24 de septiembre de 2019. Recuperado desde www.uco.es/aforac/media/recursos/Diseno_Universal_de_Aprendizaje.pdf

Duk, C., y Murillo, J. (2011). Aulas, escuelas y sistemas educativos inclusivos: la necesidad de una mirada sistémica. Revista Latinoamericana de Educación Inclusiva, 5(2), 11-12. Recuperado desde https://repositorio.uam.es/bitstream/handle/10486/662794/aulas_duk_RLEI_2011. pdf? sequence $=1$ yisAllowed $=\mathrm{y}$

Flick, U. (2004). Introducción a la investigación cualitativa. Madrid: Morata.

Fondo de las Naciones Unidas para la Infancia. (UNICEF). (2001). Inclusión de niños con discapacidad en la escuela regular. Ciclo de Debates: Desafíos de la Política Educacional, Argentina, Chile y Uruguay. Última visita 31 de mayo de 2001. Recuperado desde http:// centroderecursos.educarchile.cl/bitstream/handle/20.500.12246/2931/201304151207310.doc_ Inclusion_Unicef.pdf?sequence $=1$

Friend, M., Cook, L., Hurley-Chamberlain, D. y Shamberger, C. (2010). Co-teaching: an illustration of the complexity of collaboration in special education. Journal of Educational and Psychological Consultation, 20(1), 9-27, doi: 10.1080/10474410903535380

Fundación del Magisterio de La Araucanía. (FDMA). (2014). Proyecto educativo institucional. Última visita 20 de octubre de 2018. Recuperado desde http://www.fmda.cl/images/pdf/PEI-FMDA.pdf

Godoy, P., Meza, L. y Salazar, A. (2004). Antecedentes históricos, presente y futuro de la educación especial en chile. Santiago de Chile, Ministerio de Educación, Programa de Educación Especial. Última visita 16 de septiembre de 2019. Recuperado desde http://especial.mineduc.cl/wpcontent/uploads/sites/31/2016/08/201304151210180.doc_Antecedentes_Ed_Especial.pdf

Horn, A. \& Marfán, J. (2010). Relación entre liderazgo educativo y desempeño escolar: Revisión de la investigación en Chile. Psicoperspectivas, 9(2), 82-104. Recuperado desde: https://scielo. conicyt.cl/scielo.php?pid=S0718-69242010000200005\&script=sci_arttext\&tlng=en

Hughes, C. y Murawski, W. (2001). Lessons from another field: applying co-teaching strategies to gifted education. Journal Gifted Child Quarterly, 45(3), 195-203. Recuperado de https://doi. org/10.1177/001698620104500304

Leal-Soto, F., Albornoz, M. y Rojas, M. I. (2016). Liderazgo directivo y condiciones para la innovación en escuelas chilenas: el que nada hace, nada teme. Estudios pedagógicos (Valdivia), 42(2), 193205. Doi: https://dx.doi.org/10.4067/S0718-07052016000200011 
Leithwood, K., Day, C., Sammons, P., Harris, A. y Hopkins, D. (2006). Successful School Leadership. What it is and how it influences pupil learning. UK: National College for School Leadership.

Ministerio de Educación. (MINEDUC). (1994). Normas para la Plena Integración Social de Personas con Discapacidad (Ley $\mathrm{N}^{\circ}$ 19.284). Biblioteca del Congreso Nacional. Ley Chile. Última visita 10 de octubre de 2018. Recuperado desde https://www.leychile.cl/Navegar?idNorma=30651

. (2005). Marco para la buena dirección. Santiago de Chile: Ministerio de Educación. Última visita 12 de octubre de 2019. Recuperado desde http://archivos.agenciaeducacion.cl/Marco_ para_la_Buena_Direccion.pdf

(2009). Normas para determinar los alumnos con necesidades educativas especiales que serán beneficiarios de las subvenciones para educación especial (Decreto 170). Unidad de Educación Especial, Santiago de Chile.

(2013). Orientaciones técnicas para programas de integración escolar. División de Educación, Unidad de Educación Especial, Santiago de Chile. Última visita 12 de septiembre de 2019. Recuperado desde https:H//bibliotecadigital.mineduc.cl/handle/20.500.12365/2077

(2015a). Ley de inclusión escolar (No. 20.845). Biblioteca del Congreso Nacional. Ley Chile. Última visita 12 de septiembre de 2019. Recuperado desde https://www.google.com/url? $\mathrm{sa}=$ tyrct $=$ jyq $=$ yesrc $=$ sysource $=$ webycd $=2 \mathrm{ycad}=$ rjayuact $=8 \mathrm{yved}=2 \mathrm{ahUKEwiXwtKwnYPnAhUJ}$ KLkGHf8SDp0QFjABegQIAxAByurl=https\%3A\%2F\%2Fwww.mineduc.cl\%2Fwp-content\% 2Fuploads\%2Fsites\%2F19\%2F2018\%2F03\%2Flibro_Inclusio\%25CC\%2581n_final.pdfyusg= AOvVaw26M08N43r45KBG8HIur50D

. (2015b). Marco para la buena dirección y el liderazgo escolar. Experimentación e Investigaciones Pedagógicas (CPEIP), Santiago de Chile. Última visita 12 de noviembre de 2019. Recuperado desde http://liderazgoescolar.mineduc.cl/wp-content/uploads/sites/55/2016/04/ MBDLE_2015.pdf

. (2015c). Decreto N83: orientaciones técnicas. División de Educación, Unidad de Educación Especial, Santiago de Chile, MINEDUC. Última visita 12 de diciembre de 2019. Recuperado desde https://especial.mineduc.cl/wp-content/uploads/sites/31/2016/09/Orientaciones-PIE-2013-3.pdf

Miranda, J. (2013). Factores que obstaculizan el cambio en profesores y directivos de liceos vulnerables en la región de La Araucanía (Chile). Universidad Católica de Temuco, Chile. Recuperado desde https://www.raco.cat/index.php/Educar/article/view/287079

Muñoz, G. y Marfán, J. (2012). Formación de directores escolares en Chile. In: Weinstein, J.; Muñoz, G. ¿Qué sabemos sobre los directores de escuela en Chile? Santiago de Chile: Fundación Chile; CEPPE.

Murawski, W. (2008). Five keys to co-teaching in inclusive classrooms. School Administrator, 65(8), 29-42. Recuperado desde https://www.csun.edu/sites/default/files/Five-Keys-Co-TeachingInclusive-Classroom.pdf

Organización de las Naciones Unidas para la Educación, la Ciencia y la Cultura. (UNESCO). (1922, mayo, 2015). Declaración de Incheon. educación 2030: hacia una educación inclusiva y equitativa de calidad y un aprendizaje a lo largo de la vida para todos. Incheon, Corea: Unesco. Última visita 16 de septiembre de 2019. Recuperado desde http://unesdoc.unesco.org/ images/0023/002338/233813M.pdf

. (2001). Informe Anual del UNICEF 2001. Recuperado desde https://www.unicef.org/ spanish/publications/index_4285.html

Organización de Naciones Unidas. (ONU). (21, octubre, 2015). Transformar nuestro mundo: la Agenda 2030 para el Desarrollo Sostenible. Última visita 31 de octubre de 2019. Recuperado desde https://unctad.org/meetings/es/SessionalDocuments/ares70d1_es.pdf

Raczynski, D., Pavez, M. A., Ruiz, C., Vergara, M., Abarca, G. \& Galdames, V. (2002). Análisis de la oferta de programas e iniciativas del sector público y privado desde los establecimientos educacionales. Informe Final de Consultoría para el Ministerio de Educación. Santiago: Asesorías para el Desarrollo. 
Rodríguez, F. (2012). La percepción del trabajo colaborativo en la gestión curricular de profesores de educación regular y educación especial en programas de integración escolar de la comuna de Tomé. Tesis de maestría. Universidad del Bío-Bío, Bío-Bío, Chile. Recuperado desde http:// repobib.ubiobio.cl/jspui/bitstream/123456789/2135/1/Rodriguez_Rojas_Felipe.pdf

Rodríguez, F. (2014). La co-enseñanza, una estrategia para el mejoramiento educativo y la inclusion. Revista Latinoamericana de Educación Inclusiva, 8(2), 219-233. Recuperado desde http://www. rinace.net/rlei/numeros/vol8-num2/art11.pdf

Rodríguez, F., y Ossa, C. (2014). Valoración del trabajo colaborativo entre profesores de escuelas básicas de Tomé, Chile. Revista Estudios Pedagógicos, 40(2), 303-319. Recuperado desde https://dx.doi.org/10.4067/S0718-07052014000300018

Rubio, V. (2017). Inclusión de personas en situación de discapacidad en educación superior, desde el enfoque de la responsabilidad social, en un contexto de transiciones discursivas respecto del binomio integración/inclusión. Revista latinoamericana de educación inclusiva, 11(2), 199-216. Recuperado desde https://scielo.conicyt.cl/scielo.php?pid=S0718$73782017000200013 y$ script=sci_arttext

Sánchez, A. (2004). La educación inclusiva: dilemas y desafíos. Educación, desarrollo y diversidad, $7(2), \quad 25-40 . \quad$ Recuperado desde https://www.researchgate.net/profile/Pilar_Sanchez23/ publication/242128244_LA_EDUCACION_INCLUSIVA_DILEMAS_Y_DESAFIOS/ links/5c474759458515a4c7388f05/LA-EDUCACION-INCLUSIVA-DILEMAS-Y-DESAFIOS.pdf

Stuart, S., Connor, M., Cady, K., y Zweifel, A. (2007). Multiage instruction and inclusion: a collaborative approach. Journal International Journal of Whole Schooling, 3(1), 12-26. Recuperado desde https://eric.ed.gov/?id=EJ847473

Temprado, M. (2009). Ventajas del aprendizaje cooperativo para la socialización de los alumnos con necesidades educativas especiales. Revista Interuniversitaria de Formación del Profesorado, 23(2), 137-150. Recuperado desde http://www.redalyc.org/pdf/274/27419063010.pdf

Ulloa, J., Nail, O., Castro, A., y Muñoz, M. (2012). Problemas de gestión asociados al liderazgo como función directiva. Estudios pedagógicos (Valdivia), 38(1), 121-129. Doi: https://dx.doi. org/10.4067/S0718-07052012000100007

Vance, A. (2001). Teacher's beliefs about co-teaching. Remedial and Special Education 22(4), 245255.

Villa, R., Thousand, J., y Nevin, A. (2008). A guide to co-teaching: practical tips for facilitating student learning. Thousand Oaks: Corwin Press.

Weinstein, J. (2002) Calidad y gestión en educación: condiciones y desafíos. Pensamiento Educativo, 31(2), 50-71. Recuperado desde www.pensamientoeducativo.uc.cl/files/journals/2/articles/229/ public/229-543-1-PB.pdf

Wolfensberger, W. (1986). El debate sobre la normalización. Siglo Cero, (105), 12-28.

Zañartu, L. (2003). Aprendizaje colaborativo: una nueva forma de diálogo interpersonal $y$ en red. Recuperado desde https://tic.sepdf.gob.mx/micrositio/micrositio2/archivos/ AprendizajeColaborativo.pdf 
